\title{
Algorithm Variable Order, Step and the Configuration Variables for Solving Stiff Problems
}

\section{E. A. Novikov}

Institute of Computational Modelling, Siberian Branch of the Russian Academy of Sciences, Russia, 660036, Krasnoyarsk, Akademgorodok, novikov@icm.krasn.ru

An inequality for stability control of a Ceschino's scheme of second order of accuracy is constructed. A numerical formula of order one is developed that is based on the stages of the this method and its stability interval is extended to 32 . On a base of L-stable (2,1)-scheme and a numerical Ceschino's formula, an algorithm of alternating structure, in which an efficient numerical formula is chosen on an every step by a stability criterion, is constructed. The algorithm is intended for solving stiff and non-stiff problems. There are shown results of calculations, confirming efficiency of this algorithm.

Key words: stiff problem, Ceschino's scheme, (2,1)-method, accuracy and stability control.

\section{References}

1. Hairer E., Wanner G. Solving ordinary differential equations II. Stiff and differential-Algebraic problems. New York, Springer-Verlag, 1996, 601 p.

2. Byrne G. D., Hindmarsh A. C. ODE solvers : a review of current and coming attractions. J. of Comput. Physics, 1987, no. 70, pp. 1-62.

3. Rosenbrock H. H. Some general implicit processes for the numerical solution of differential equations. Computer, 1963, no. 5, pp. 329-330.

4. Novikov V. A., Novikov E. A., Yumatova L. A. Freezing of a matrix of Jacobi in the Rosenbrock method of the second order of accuracy. Zhurnal Vychislitel'noi Matematiki i Matematicheskoi Fiziki, 1987, vol. 27, no. 3, pp. 385-390 (in Russian).

5. Novikov E. A. Construction of algorithm for the integrating stiff differential equations on nonuniform schemes. Soviet Math. Dokl., 1984, vol. 30, no. 2, pp. 358361 .

6. Novikov E. A. Algorithm of Integrating Stiff Problems Using the Explicit and Implicit Methods. Izv. Sarat. Univ. N.S. Ser. Math. Mech. Inform., 2012, vol. 12, no. 4, pp.19-27 (in Russian).

7. Novikov V. A., Novikov E. A. Increase of efficiency of algorithms of integration of the ordinary differential equations at the expense of stability control. Zhurnal Vychislitel'noi Matematiki $i$ Matematicheskoi Fiziki, 1985, vol. 25, no. 7, pp. 1023-1030 (in Russian).

8. Novikov E. A. Explicit methods for stiff systems. Novosibirsk, Nauka, 1997, 197 p. (in Russian).

9. Novikov E. A., Shornikov Yu. V. Computer modeling of stiff hybrid systems. Novosibirsk, publishing house NGTU, 2012, 450 p. (in Russian).

10. Novikov E. A., Shitov Yu. A., Shokin Yu. I. Onestep iteration-free methods of solving stiff systems. Soviet Math. Dokl., 1989, vol. 38, no. 1, pp. 212-216.

11. Novikov A. E., Novikov E. A. Numerical integration of stiff systems with low accuracy. Mathematical Models and Computer Simulations, 2010, vol. 2, no. 4, pp. 443452. DOI: $10.1134 / \mathrm{S} 2070048210040046$.

12. Ceschino F., Kuntzman J. Numerical solution of initial value problems. New Jersey: Prentice-Hall, Englewood Clis, 1966, 287 p.

13. Hindmarsh A. C. ODEPACK, a systematized collection of ODE solvers. Lawrence Livermore National Laboratory, 1982, preprint UCRL-88007.

УДК 514.133

\section{ГИПЕРБОЛИЧЕСКИЕ ПАРАЛЛЕЛОГРАММЫ ПЛОСКОСТИ $\widehat{H}$}

\section{Л. Н. Ромакина}

Кандидат фризико-математических наук, доцент касредры геометрии, Саратовский государственный университет им. Н. Г. Чернышевского, romakinaln@mail.ru

На гиперболической плоскости $\widehat{H}$ положительной кривизны в модели Кэли -Клейна исследованы гиперболические параллелограммы. Проведена их классисрикация, получены метрические соотношения между величинами углов и выражения длин ребер через меры углов при вершинах.

Ключевые слова: гиперболическая плоскость $\widehat{H}$ положительной кривизны; параллелограмм; гиперболический параллелограмм.

\section{ВВЕДЕНИЕ}

Гиперболическую плоскость $\widehat{H}$ положительной кривизны [1-5] рассматриваем в проективной интерпретации Кэли-Клейна как внешнюю относительно овальной линии $\gamma$, называемой абсолютом плоскости $\widehat{H}$, область проективной плоскости $P_{2}$. 
Наличие на $\widehat{H}$ двух типов параллельности позволяет выделить три типа параллелограммов этой плоскости. В данной работе исследованы гиперболические параллелограммы, все стороны которых являются гиперболическими прямыми. По типу расположения на абсолюте точек сторон проведена классификация параллелограммов. Используемый метод классификации предложен в работах [6,7] при исследовании $n$-контуров. Показано, что различные типы положений на абсолюте точек сторон определяют на $\widehat{H}$ четыре класса гиперболических параллелограммов. Для параллелограммов каждого класса получены: соотношения между длинами ребер и между внутренними и внешними углами при вершинах; выражения длин ребер через величины гиперболических углов (внешних или внутренних) при вершинах соответствующих ребер.

Приведем используемые в работе основные понятия и формулы.

При выводе метрических формул используем только вещественные координаты действительных точек и прямых в каноническом репере $R$ второго типа [3]. Уравнение абсолюта в репере $R$ в координатах текущей точки (прямой) имеет вид

$$
x_{1} x_{2}-x_{3}^{2}=0 \quad\left(4 X_{1} X_{2}-X_{3}^{2}=0\right) .
$$

Для координат $\left(m_{i}\right), i=1,2,3$, собственной для $\widehat{H}$ точки $M$ в репере $R$ выполняется неравенство

$$
m_{1} m_{2}-m_{3}^{2}<0
$$

Значение квадратичной формы $\Phi=4 X_{1} X_{2}-X_{3}^{2}$ от координат $\left(a_{i}\right)$ действительной прямой $a$ назовем характеристикой данных координат прямой в заданном репере: $\Phi_{a}\left(a_{i}\right)=4 a_{1} a_{2}-a_{3}^{2}$. Если в репере $R$ координаты $\left(a_{i}\right)$ и $\left(b_{i}\right)$ задают одну прямую $a$, то согласно определению проективных координат существует $\lambda \neq 0$ такое, что для всех индексов $i$ выполняется равенство $b_{i}=\lambda a_{i}$. Следовательно, характеристики $\Phi_{a}\left(a_{i}\right), \Phi_{a}\left(b_{i}\right)$ координат $\left(a_{i}\right)$ и $\left(b_{i}\right)$ прямой $a$ в репере $R$ связаны условием $\Phi_{a}\left(b_{i}\right)=\lambda^{2} \Phi_{a}\left(a_{i}\right)$ и являются вещественными числами одного знака. Каждая характеристика координат эллиптической, гиперболической и параболической прямой $a$ в репере $R$ удовлетворяет соответственно условию

$$
\Phi_{a}>0, \quad \Phi_{a}<0, \quad \Phi_{a}=0
$$

Пара собственных для $\widehat{H}$ точек гиперболической или параболической прямой определяет на этой прямой отрезок и пару лучей. Отрезки гиперболических прямых являются измеримыми на $\widehat{H}$. Если собственные для плоскости $\widehat{H}$ точки $A, B$ принадлежат гиперболической прямой, то прямая $A B$ содержит две действительные точки абсолютной линии $\gamma$, обозначим их как $K_{1}, K_{2}$. Сложное отношение $\left(A B K_{1} K_{2}\right)$ четверки точек прямой является инвариантом группы $G$. Число $|A B|=\frac{\rho}{2}\left|\ln \left(A B K_{1} K_{2}\right)\right|$, где $\rho, \rho \in \mathbb{R}_{+},-$радиус кривизны плоскости $\widehat{H}$, назовем расстоянием между точками $A, B$ и длиной отрезка $A B$.

Расстояние между точками $A\left(a_{i}\right), B\left(b_{i}\right), i=1,2,3$, гиперболической прямой в репере $R$ можно вычислить по формуле

$$
\operatorname{ch} \frac{|A B|}{\rho}= \pm \frac{a_{1} b_{2}+a_{2} b_{1}-2 a_{3} b_{3}}{2 \sqrt{a_{1} a_{2}-a_{3}^{2}} \sqrt{b_{1} b_{2}-b_{3}^{2}}} .
$$

Пучок прямых плоскости $\widehat{H}$ назовем гиперболическим (эллиптическим), если его центр - внешняя (внутренняя) относительно абсолюта точка. Параболическим пучком назовем пучок с центром на абсолюте. Прямые, принадлежащие гиперболическому (эллиптическому) пучку, назовем пересекающимися (расходящимися) на $\widehat{H}$. Прямые параболического пучка назовем параллельными.

Пара прямых на плоскости $\widehat{H}$ в зависимости от типов прямых в паре и от типа содержащего данные прямые пучка может определять 15 типов углов плоскости $\widehat{H}$ [4]. Определим типы углов, используемых в данной работе.

Две гиперболические пересекающиеся прямые $a$ и $b$ разбивают плоскость $\widehat{H}$ на два вертикальных гиперболических угла, симметричных относительно общей точки прямых $a, b$, и смежный с каждым из этих углов гиперболический псевдоугол.

Параллельные гиперболические прямые $a$ и $b$ определяют две связные области на $\widehat{H}$. Ту область, которая не содержит (содержит) поляру общей точки прямых $a$ и $b$ относительно абсолюта назовем полосой (псевдополосой) плоскости $\widehat{H}$ между прямыми $a$ и $b$. 
Гиперболическая $a$ и эллиптическая $b$ прямые разбивают плоскость $\widehat{H}$ на две связные части. Каждую из них назовем квазиуглом между прямыми $a, b$. По отношению друг к другу эти части назовем смежньми.

Пусть гиперболическая $a$ и эллиптическая $b$ прямые образуют смежные квазиуглы $\nu_{1}, \nu_{2}$. Пара прямых $a, b$ в пучке с центром во внешней относительно абсолюта точке $K$ разделяет пару действительных абсолютных касательных $k_{1}, k_{2}$. Следовательно, $\left(a b k_{1} k_{2}\right) \in \mathbb{R}_{-}$. Тогда выполняются равенства

$$
\ln \left(a b k_{1} k_{2}\right)=\pi i+\ln \left|\left(a b k_{1} k_{2}\right)\right|, \quad \ln \left(b a k_{1} k_{2}\right)=\pi i-\ln \left|\left(a b k_{1} k_{2}\right)\right|,
$$

где функция $w=\ln z-$ главное значение логарифмической функции $w=\operatorname{Ln} z$ :

$$
\ln z=\ln |z|+i \arg z, \quad-\pi<\arg z \leq \pi
$$

Числа $\left[\pi i \pm \ln \left|\left(a b k_{1} k_{2}\right)\right|\right] / 2$, где $\ln \left|\left(a b k_{1} k_{2}\right)\right| \in \mathbb{R}$, назовем мерами или величинами квазиуглов $\nu_{1}$, $\nu_{2}$.

Если прямые $a, b$ гармонически разделяют пару $k_{1}, k_{2}$, т.е. $\left(a b k_{1} k_{2}\right)=-1$, то мера квазиугла между ними равна $\pi i / 2$. Прямые $a, b$ в этом случае назовем ортогональными. Обозначение: $a \perp b$.

Пусть пересекающиеся гиперболические прямые $a, b$ образуют вертикальные гиперболические углы $\nu_{1}, \nu_{2}$ и смежный с каждым из этих углов гиперболический псевдоугол $\psi$. Пара прямых $a, b$ в пучке с центром в собственной для $\widehat{H}$ точке $K$ не разделяет пары абсолютных касательных $k_{1}, k_{2}$, т. е. $\left(a b k_{1} k_{2}\right) \in \mathbb{R}_{+}$.

Число $v=\frac{1}{2}\left|\ln \left(a b k_{1} k_{2}\right)\right|, v \in \mathbb{R}_{+}$, назовем мерой или величиной гиперболического угла $\nu_{1}\left(\nu_{2}\right)$.

Если непараболические прямые $a, b$ принадлежат параболическому пучку, то касательные к абсолюту, проведенные через точку $K$, совпадают: $k_{1}=k_{2}$. В этом случае $\left(a b k_{1} k_{2}\right)=1, \ln \left(a b k_{1} k_{2}\right)=0$, т. е. вычисленная формально мера полосы (псевдополосы), определенной параллельными гиперболическими прямыми, равна нулю. Следовательно, пара параллельных гиперболических прямых не имеет инварианта относительно группы $G$.

Меру $\widehat{a b}$ угла между неизотропными пересекающимися прямыми $a\left(a_{i}\right), b\left(b_{i}\right), i=1,2,3$, в репере $R$ можно выразить формулой

$$
\operatorname{ch} \widehat{a b}= \pm \frac{2 a_{1} b_{2}+2 a_{2} b_{1}-a_{3} b_{3}}{\sqrt{4 a_{1} a_{2}-a_{3}^{2}} \sqrt{4 b_{1} b_{2}-b_{3}^{2}}} .
$$

\section{2. КЛАССИФИКАЦИЯ ГИПЕРБОЛИЧЕСКИХ ПАРАЛЛЕЛОГРАММОВ}

Четырехвершинником плоскости $\widehat{H}$ назовем совокупность четырех точек, никакие три из которых не лежат на одной прямой, и четырех отрезков (эллиптических, гиперболических или параболических прямых), циклически соединяющих эти точки. Данные точки назовем вершинами, отрезки ребрами, а прямые, содержащие данные отрезки, - сторонами четырехвершинника.

Ребра (стороны), имеющие общую вершину, назовем смежными ребрами (сторонами) четырехвершинника. Ребра (стороны), не являющиеся смежными, назовем противоположными.

Вершины, принадлежащие (не принадлежищие) одному ребру, назовем смежными (противоположными) вершинами четырехвершинника. Прямые, проходящие через противоположные вершины четырехвершинника, назовем диагональными прямыми данного четырехвершинника.

В данной работе условимся рассматривать только конечные четырехвершинники, все точки которых являются собственными на $\widehat{H}$.

Параллелограммом плоскости $\widehat{H}$ назовем четырехвершинник, противоположные стороны которого параллельны.

Каждый угол между смежными сторонами параллелограмма будем называть углом при вершине данных сторон, указывая при необходимости его тип. Угол при вершине параллеограмма назовем внутренним, если он содержит противоположную вершину параллелограмма. Угол, смежный с внутренним углом при вершине, назовем внешним углом параллелограмма при данной вершине.

Параллельными в паре на $\widehat{H}$ могут быть либо две гиперболические прямые, либо гиперболическая и параболическая прямые. Поэтому все параллелограммы плоскости $\widehat{H}$ можно отнести к трем типам. Гиперболическим назовем параллелограмм, все стороны которого гиперболические, параболическим - параллелограмм, содержащий одну параболическую сторону. Параллелограмм с двумя параболическими сторонами назовем бипараболическим. 
Классификацию гиперболических параллелограммов проведем, учитывая расположение на абсолюте несобственных точек сторон.

Все стороны гиперболического параллелограмма содержат шесть точек абсолюта, причем две из этих точек, обозначим их через $Q, S$ - точки пересечения противоположных сторон. Все вершины конечного параллелограмма - собственные точки плоскости $\widehat{H}$, поэтому внутренние относительно $\gamma$ хорды сторон параллелограмма не имеют общих внутренних относительно $\gamma$ точек. Следовательно, с точностью до обозначения в парах противоположных сторон возможны лишь четыре типа расположения на абсолютной линии $\gamma$ точек сторон параллелограмма:

1) точки $Q, S$ разбивают $\gamma$ на две дуги, одна из которых содержит концы хорд с общей точкой $Q$, другая - концы хорд с общей точкой $S$ (рис. $1, a)$;

2) точки $Q, S$ разбивают $\gamma$ на две дуги, каждая из которых содержит концы одной из хорд, исходящих из $Q$, и одной из хорд, исходящих из $S$ (рис. $1, \sigma)$;

3) точки $Q, S$ разбивают линию $\gamma$ на две дуги, одна из которых не содержит точек сторон параллелограмма (рис. 1, в);

$4)$ одна из дуг линии $\gamma$, образованных точками $Q, S$, содержит одну из четырех точек сторон параллелограмма на абсолюте (рис. 1, г).

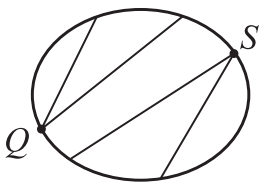

$a$

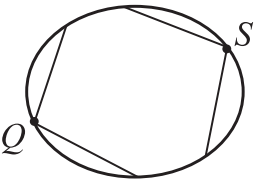

б

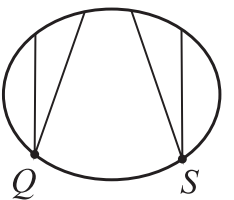

B

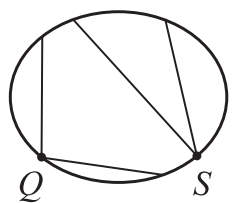

2

Рис. 1. Типы расположений на абсолютной овальной линии $\gamma$ точек сторон гиперболического параллелограмма

Соответственно типу 1)-4) расположения точек сторон на абсолюте гиперболический параллелограмм отнесем к классу $h(I), h(I I), h(I I I), h(I V)$.

\section{3. СВОЙСТВА ГИПЕРБОЛИЧЕСКИХ ПАРАЛЛЕЛОГРАММОВ}

Для гиперболического параллелограмма $A B C D$ введем обозначения: $A B=a, B C=b, C D=c$, $D A=d, a \cap \gamma=\left\{A_{0}, Q\right\}, b \cap \gamma=\left\{B_{0}, S\right\}, c \cap \gamma=\left\{C_{0}, Q\right\}, d \cap \gamma=\left\{D_{0}, S\right\}$. Величину угла при вершине параллелограмма будем обозначать той же буквой, что и вершину, причем величину внешнего угла будем помечать чертой сверху. Обозначать вершины параллелограмма $A B C D$ (рис. 2,3 ) условимся так, чтобы правильно расположенными на $\gamma$ [7] соответственно классу параллелограмма были точки упорядоченной системы:

$$
\begin{array}{llll}
h(I): & T_{1}=\left\{Q, C_{0}, A_{0}, S, B_{0}, D_{0}\right\} ; & h(I I): & T_{2}=\left\{Q, A_{0}, B_{0}, S, D_{0}, C_{0}\right\} ; \\
h(I I I): & T_{3}=\left\{Q, C_{0}, A_{0}, B_{0}, D_{0}, S\right\} ; & h(I V): & T_{4}=\left\{Q, C_{0}, A_{0}, B_{0}, S, D_{0}\right\} .
\end{array}
$$

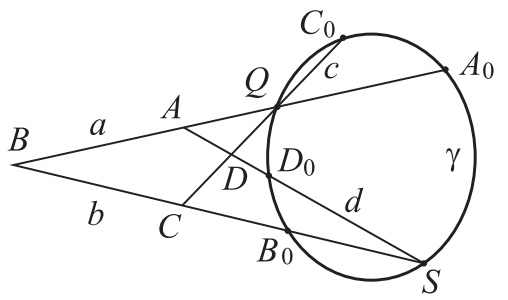

$a$

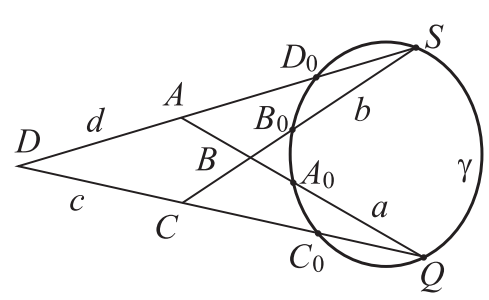

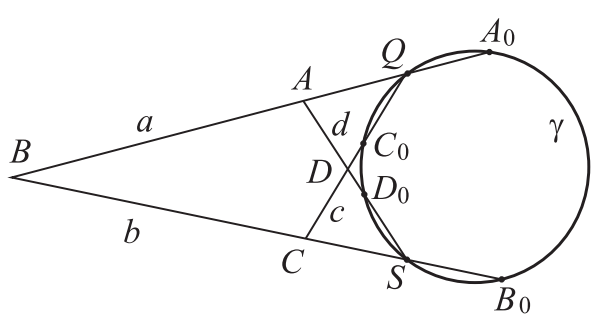

б

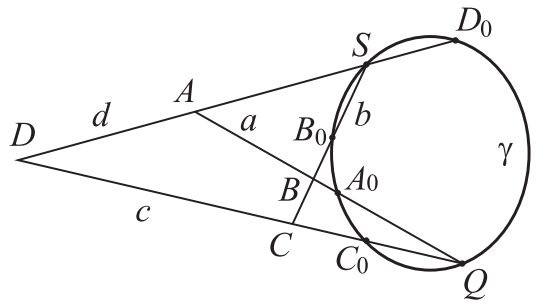

2

Рис. 2. Гиперболические параллелограммы класса: $h(I)(a) ; h(I I)(б), h(I I I)($ в); $h(I V)($ () 
Каждые две смежные стороны гиперболического параллелограмма образуют два вертикальных гиперболических угла и смежный с ними гиперболический псевдоугол. В метрические соотношения будем включать только меры гиперболических углов при вершинах параллелограммов, внутренних или внешних, учитывая, что они являются действительными положительными числами.

Теорема. Пусть АВCD - гиперболический параллелограмм плоскости $\widehat{H}$ действительного радиуса кривизны $\rho$, точки соответствующей системы (6) которого правильно расположень на $\gamma$. Справедливы следующие утверждения.

1. Диагональная прямая ВD в гиперболическом параллелограмме каждого класса является гиперболической. Диагональная прямая AC параллелограмма класса $h(I I)$ является эллиптической. В параллелограмме класса $h(I), h(I I I), h(I V)$ диагональная прямая АC может быть любого muna.

2. Внутренние углы параллелограмма при вершинах $A, C(B, D)$ являются гиперболическими псевдоуглами (углами).

3. Соответственно классу параллелограмма величины гиперболических углов при вершинах внешних $\bar{A}, \bar{C}$ и внутренних $B, D$ связань следующими условиями:

$$
\begin{aligned}
& h(I): \quad \operatorname{sh} \frac{\bar{A}}{2} \operatorname{sh} \frac{\bar{C}}{2}=\operatorname{sh} \frac{B}{2} \operatorname{sh} \frac{D}{2}, \quad \bar{A}<B<\bar{C}, \quad \bar{A}<D<\bar{C} \text {; } \\
& h(I I): \quad \operatorname{sh} \frac{\bar{A}}{2} \operatorname{sh} \frac{\bar{C}}{2}=\operatorname{ch} \frac{B}{2} \operatorname{ch} \frac{D}{2} \\
& h(I I I): \quad \operatorname{ch} \frac{\bar{A}}{2} \operatorname{ch} \frac{\bar{C}}{2}=\operatorname{ch} \frac{B}{2} \operatorname{ch} \frac{D}{2}, \quad B<\bar{A}<D, \quad B<\bar{C}<D \text {; } \\
& h(I V): \quad \operatorname{sh} \frac{\bar{A}}{2} \operatorname{ch} \frac{\bar{C}}{2}=\operatorname{ch} \frac{B}{2} \operatorname{sh} \frac{D}{2}, \quad B<\bar{C}, \quad \bar{A}<D .
\end{aligned}
$$

4. Соответственно классу параллелограмма длины его ребер зависят от мер гиперболических углов при вершинах следующим образом:

$$
\begin{aligned}
& h(I): \quad|A B|=\rho \ln \left(\operatorname{th} \frac{B}{2} \operatorname{cth} \frac{\bar{A}}{2}\right), \quad|B C|=\rho \ln \left(\operatorname{th} \frac{\bar{C}}{2} \operatorname{cth} \frac{B}{2}\right), \\
& |C D|=\rho \ln \left(\operatorname{th} \frac{\bar{C}}{2} \operatorname{cth} \frac{D}{2}\right), \quad|A D|=\rho \ln \left(\operatorname{th} \frac{D}{2} \operatorname{cth} \frac{\bar{A}}{2}\right) ; \\
& h(I I): \quad|A B|=\rho \ln \left(\operatorname{cth} \frac{\bar{A}}{2} \operatorname{cth} \frac{B}{2}\right), \quad|B C|=\rho \ln \left(\operatorname{cth} \frac{B}{2} \operatorname{cth} \frac{\bar{C}}{2}\right) \text {, } \\
& |C D|=\rho \ln \left(\operatorname{cth} \frac{\bar{C}}{2} \operatorname{cth} \frac{D}{2}\right), \quad|A D|=\rho \ln \left(\operatorname{cth} \frac{\bar{A}}{2} \operatorname{cth} \frac{D}{2}\right) ; \\
& h(I I I): \quad|A B|=\rho \ln \left(\operatorname{th} \frac{\bar{A}}{2} \operatorname{cth} \frac{B}{2}\right), \quad|B C|=\rho \ln \left(\operatorname{th} \frac{\bar{C}}{2} \operatorname{cth} \frac{B}{2}\right) \text {, } \\
& |C D|=\rho \ln \left(\operatorname{th} \frac{D}{2} \operatorname{cth} \frac{\bar{C}}{2}\right), \quad|A D|=\rho \ln \left(\operatorname{th} \frac{D}{2} \operatorname{cth} \frac{\bar{A}}{2}\right) ; \\
& h(I V): \quad|A B|=\rho \ln \left(\operatorname{cth} \frac{\bar{A}}{2} \operatorname{cth} \frac{B}{2}\right), \quad|B C|=\rho \ln \left(\operatorname{th} \frac{\bar{C}}{2} \operatorname{cth} \frac{B}{2}\right), \\
& |C D|=\rho \ln \left(\operatorname{cth} \frac{\bar{C}}{2} \operatorname{cth} \frac{D}{2}\right), \quad|A D|=\rho \ln \left(\operatorname{th} \frac{D}{2} \operatorname{cth} \frac{\bar{A}}{2}\right) \text {. }
\end{aligned}
$$

Доказательство. Присоединим к гиперболическому параллелограмму $A B C D$ канонический репер $R=\left\{Q, S, A_{3}, A_{0}\right\}$ второго типа, где $A_{3}-$ полюс прямой $Q S$ относительно абсолюта. Найдем координаты точек системы (6), вершин, сторон и диагональных прямых параллелограмма $A B C D$ в репере $R$.

Точки $A, B$ прямой $a=Q A_{0}(0: 1:-1)$ можно задать координатами: $A(u: 1: 1), B(v: 1: 1)$, $u, v \in \mathbb{R}$. Точка $C$ принадлежит прямой $b=S B(1: 0:-v)$. Присвоим точке $C$ координаты $(v: t: 1)$, где $t \in \mathbb{R}$. Тогда точка $D$ пересечения прямых $d=A S(1: 0:-u), c=C Q(0: 1:-t)$ имеет в $R$ координаты $(u: t: 1)$. Итак,

$$
\begin{array}{crrl}
Q(1: 0: 0), & S(0: 1: 0), & A_{0}(1: 1: 1), & A(u: 1: 1), \\
B(v: 1: 1), & C(v: t: 1), & D(u: t: 1), & t, u, v \in \mathbb{R} ;
\end{array}
$$




$$
\begin{array}{rrrr}
a(0: 1:-1), & b(1: 0:-v), & c(0: 1:-t), \quad d(1: 0:-u), \\
A C(1-t: v-u: t u-v), & B D(1-t: u-v: t v-u) .
\end{array}
$$

Вершины параллелограмма являются собственными на $\widehat{H}$ точками, поэтому их координаты из (15) в силу неравенства (2) удовлетворяют условиям:

$$
u<1, \quad v<1, \quad t u<1, \quad t v<1 .
$$

Точки абсолюта (1) на сторонах параллелограмма, не входящие в репер $R$, имеют координаты

$$
B_{0}\left(v^{2}: 1: v\right), \quad C_{0}\left(1: t^{2}: t\right), \quad D_{0}\left(u^{2}: 1: u\right) .
$$

Потребуются также координаты прямых

$$
S A_{0}(1: 0:-1), \quad S C_{0}(t: 0:-1), \quad Q B_{0}(0: v:-1), \quad Q D_{0}(0: u:-1) .
$$

Определим допустимые значения параметров $t, u, v$, при которых параллелограмм $A B C D$ относится к классу $h(I), h(I I), h(I I I), h(I V)$.

Положение на абсолюте точек системы (6) можно характеризовать числами:

$$
\begin{gathered}
I_{1}=\left(\left(S A_{0}\right) b\left(S C_{0}\right)(S Q)\right), \quad I_{2}=\left(d(S Q) b\left(S A_{0}\right)\right)=\left(A Q B A_{0}\right), \quad I_{3}=\left(a(Q S) c\left(Q B_{0}\right)\right)=\left(B S C B_{0}\right), \\
I_{4}=\left(b(S Q) d\left(S C_{0}\right)\right)=\left(C Q D C_{0}\right), \quad I_{5}=\left(c(Q S) a\left(Q D_{0}\right)\right)=\left(D S A D_{0}\right) .
\end{gathered}
$$

Для гиперболических параллелограммов соответственно их классу в принятых обозначениях получаем следующие неравенства для чисел (20):

$$
\begin{aligned}
& h(I): \quad I_{1}>0, \quad I_{2}>0, \quad I_{3}>0, \quad I_{4}<0, \quad I_{5}<0 ; \\
& h(I I): \quad I_{1}>0, \quad I_{2}>0, \quad I_{3}<0, \quad I_{4}>0, \quad I_{5}<0 \text {; } \\
& h(I I I): \quad I_{1}>0, \quad I_{2}>0, \quad I_{3}<0, \quad I_{4}<0, \quad I_{5}>0 \\
& h(I V): \quad I_{1}>0, \quad I_{2}>0, \quad I_{3}<0, \quad I_{4}<0, \quad I_{5}<0 .
\end{aligned}
$$

В репере $R$ числа (20) в координатах (15), (16), (18), (19) имеют выражения

$$
I_{1}=\frac{t-1}{t v-1}, \quad I_{2}=\frac{v-u}{1-u}, \quad I_{3}=\frac{v(1-t)}{v-1}, \quad I_{4}=\frac{t(v-u)}{t v-1}, \quad I_{5}=\frac{u(t-1)}{t u-1} .
$$

С учетом неравенств (17), (21) и выражений (22) получаем допустимые значения параметров $t, u, v$ координат в присоединенном репере $R$ вершин гиперболических параллелограммов соответствующего класса:

$$
\begin{aligned}
& h(I): \quad t \in(0 ; 1), \quad u<0, \quad v<0, \quad u<v \text {; } \\
& h(I I): \quad t<0, \quad u<0, \quad v \in(0 ; 1), \quad t u<1 \text {; } \\
& h(I I I): \quad t \in(0 ; 1), \quad u \in(0 ; 1), \quad v \in(0 ; 1), \quad u<v \text {; } \\
& h(I V): \quad t \in(0,1), \quad u<0, \quad v \in(0 ; 1) .
\end{aligned}
$$

При любых значениях (23) параметров $t, u, v$ характеристика координат прямой $B D(16)$ гиперболического параллелограмма каждого класса меньше нуля: $\Phi_{B D}=-4(1-t)(v-u)-(u-t v)^{2}<0$, следовательно, в гиперболическом параллелограмме любого класса диагональная прямая $B D$ является гиперболической.

Для координат (16) диагональной прямой $A C: \Phi_{A C}=4(1-t)(v-u)-(t u-v)^{2}$.

При любых значениях (23) параметров $t, u, v$ в параллелограмме класса $h(I I)$ :

$$
\Phi_{A C}=4(1-t)(v-u)-(t u-v)^{2}=v(4-v)+t u(4-t u)+u(t v-4)+t v(u-4)>0 .
$$

Следовательно, диагональная прямая $A C$ в гиперболическом параллелограмме класса $h(I I)$ является эллиптической.

Для параллелограмма класса $h(I), h(I I I), h(I V)$ зафиксируем по три набора $(t ; u ; v)$ параметров из допустимых значений (23):

$$
\begin{array}{llll}
h(I): & \left(\frac{1}{3} ;-3 ;-1\right), & \left(\frac{8}{9} ;-3 ;-1\right), & \left(\frac{7}{9} ;-3 ;-1\right) ; \\
h(I I I): & \left(\frac{1}{2} ; \frac{1}{4} ; \frac{1}{2}\right), & \left(\frac{19}{20} ; \frac{1}{4} ; \frac{1}{2}\right), & \left(4 \sqrt{3}-6 ; \frac{1}{4} ; \frac{1}{2}\right) ; \\
h(I V): & \left(\frac{1}{2} ;-1 ; \frac{1}{2}\right), & \left(\frac{4}{5} ;-1 ; \frac{1}{2}\right), & \left(3 \sqrt{2}-\frac{7}{2} ;-1 ; \frac{1}{2}\right) .
\end{array}
$$


Характеристики координат (16) прямой $A C$ при наборах из (24) равны:

$$
\begin{array}{llll}
h(I): & \Phi_{A C}=12, & \Phi_{A C}=-153, & \Phi_{A C}=0 \\
h(I I I): & \Phi_{A C}=23, & \Phi_{A C}=-121, & \Phi_{A C}=0 \\
h(I V): & \Phi_{A C}=8, & \Phi_{A C}=-49, & \Phi_{A C}=0 .
\end{array}
$$

Наборы (25) характеристик координат (16) прямой $A C$ согласно условиям (3) доказывают, что в гиперболическом параллелограмме класса $h(I), h(I I I), h(I V)$ диагональная прямая $A C$ может быть любого типа. Утверждение 1 доказано.

Чтобы доказать второе утверждение теоремы, построим параболические прямые $k_{A}, k_{B}, k_{C}, k_{D}$, проходящие через соответствующие вершины параллелограмма. Характеристики координат параболической прямой раны нулю, поэтому в репере $R$ одна из двух касательных к абсолюту, проходящих через каждую вершину параллелограмма, может быть задана соответственно координатами:

$$
\begin{array}{cc}
k_{A}\left((\sqrt{1-u}-1)^{2}: 1: 2(\sqrt{1-u}-1)\right), & k_{B}\left((\sqrt{1-v}-1)^{2}: 1: 2(\sqrt{1-v}-1)\right) \\
k_{C}\left((\sqrt{1-t v}-1)^{2}: v^{2}: 2 v(\sqrt{1-t v}-1)\right), & k_{D}\left((\sqrt{1-t u}-1)^{2}: u^{2}: 2 u(\sqrt{1-t u}-1)\right) .
\end{array}
$$

На основании условий (23), применяя координаты (16), (26), находим:

$$
\begin{aligned}
& J_{1}=\left(a d(A C) k_{A}\right)=\frac{(1-t)(v-u)^{-1}}{(\sqrt{1-u}-1)^{2}}>0, \quad J_{2}=\left(c b(A C) k_{C}\right)=\frac{v^{2}(1-t)(v-u)^{-1}}{(\sqrt{1-t v}-1)^{2}}>0 \\
& J_{3}=\left(a b(B D) k_{B}\right)=\frac{(1-t)(u-v)^{-1}}{(\sqrt{1-v}-1)^{2}}<0, \quad J_{4}=\left(c d(B D) k_{D}\right)=\frac{v^{2}(1-t)(u-v)^{-1}}{(\sqrt{1-t u}-1)^{2}}<0 .
\end{aligned}
$$

Неравенства (27) означают следующее. Прямая $A C$ не разделяет с прямой $k_{A}\left(k_{C}\right)$ пару прямых $a, d(c, b)$, так как $J_{1}>0\left(J_{2}>0\right)$. Поэтому прямая $A C$ принадлежит полностью и гиперболическому псевдоуглу между прямыми $a, d$, и гиперболическому псевдоуглу между прямыми $b, c$. Следовательно, внутренние углы параллелограмма $A B C D$ при вершинах $A, C$ являются гиперболическими псевдоуглами. Прямая $B D$ разделяет с прямой $k_{B}\left(k_{D}\right)$ пару прямых $a, b(c, d)$, так как $J_{3}<0\left(J_{4}<0\right)$. Поэтому прямая $B D$ принадлежит полностью и объединению гиперболических углов между прямыми $a, b$, и объединению гиперболических углов между прямыми $c, d$. Следовательно, внутренние углы параллелограмма $A B C D$ при вершинах $B, D$ являются гиперболическими углами. Второе утверждение теоремы доказано.

По формуле (5) выразим через параметры $t, u, v$ величины гиперболических углов, внешних $\bar{A}, \bar{C}$ и внутренних $B, D$, параллелограмма $A B C D$ при его вершинах:

$\operatorname{ch} \bar{A}=\epsilon_{1} \frac{2-u}{u}, \quad \operatorname{ch} B=\epsilon_{2} \frac{2-v}{v}, \quad \operatorname{ch} \bar{C}=\epsilon_{3} \frac{2-t v}{t v}, \quad \operatorname{ch} D=\epsilon_{4} \frac{2-t u}{t u}, \quad \epsilon_{i}= \pm 1, \quad i=\overline{1,4}$.

Учитывая, что $\bar{A} \in \mathbb{R}_{+}, B \in \mathbb{R}_{+}, \bar{C} \in \mathbb{R}_{+}, D \in \mathbb{R}_{+}$, согласно (23) определим числа $\epsilon_{i}$ из выражений (28). Соответственно классу параллелограмма получаем:

$$
\begin{aligned}
& h(I) \quad: \quad \epsilon_{1}=-1, \quad \epsilon_{2}=-1, \quad \epsilon_{3}=-1 \quad \epsilon_{4}=-1 \text {; } \\
& h(I I) \quad: \quad \epsilon_{1}=-1, \quad \epsilon_{2}=1, \quad \epsilon_{3}=-1 \quad \epsilon_{4}=1 ; \\
& h(I I I) \quad: \quad \epsilon_{1}=1, \quad \epsilon_{2}=1, \quad \epsilon_{3}=1 \quad \epsilon_{4}=1 ; \\
& h(I V): \epsilon_{1}=-1, \quad \epsilon_{2}=1, \quad \epsilon_{3}=1 \quad \epsilon_{4}=-1 \text {. }
\end{aligned}
$$

Из равенств (28) при соответствующих значениях чисел $\epsilon_{i}(29)$ для параллелограмма заданного класса находим:

$$
\begin{aligned}
& h(I): \quad u=\frac{2}{1-\operatorname{ch} \bar{A}}, \quad v=\frac{2}{1-\operatorname{ch} B}, \quad t v=\frac{2}{1-\operatorname{ch} \bar{C}}, \quad t u=\frac{2}{1-\operatorname{ch} D} \\
& h(I I): \quad u=\frac{2}{1-\operatorname{ch} \bar{A}}, \quad v=\frac{2}{1+\operatorname{ch} B}, \quad t v=\frac{2}{1-\operatorname{ch} \bar{C}}, \quad t u=\frac{2}{1+\operatorname{ch} D} \\
& h(I I I): \quad u=\frac{2}{1+\operatorname{ch} \bar{A}}, \quad v=\frac{2}{1+\operatorname{ch} B}, \quad t v=\frac{2}{1+\operatorname{ch} \bar{C}}, \quad t u=\frac{2}{1+\operatorname{ch} D} \\
& h(I V): \quad u=\frac{2}{1-\operatorname{ch} \bar{A}}, \quad v=\frac{2}{1+\operatorname{ch} B}, \quad t v=\frac{2}{1+\operatorname{ch} \bar{C}}, \quad t u=\frac{2}{1-\operatorname{ch} D} .
\end{aligned}
$$


Исключая из равенств (30)-(33) параметры $t, u, v$, получаем соотношения

$$
\begin{array}{lc}
h(I): \quad(\operatorname{ch} \bar{A}-1)(\operatorname{ch} \bar{C}-1)=(\operatorname{ch} B-1)(\operatorname{ch} D-1), \\
h(I I): \quad(\operatorname{ch} \bar{A}-1)(\operatorname{ch} \bar{C}-1)=(\operatorname{ch} B+1)(\operatorname{ch} D+1), \\
h(I I I): \quad(\operatorname{ch} \bar{A}+1)(\operatorname{ch} \bar{C}+1)=(\operatorname{ch} B+1)(\operatorname{ch} D+1), \\
h(I V): \quad(\operatorname{ch} \bar{A}-1)(\operatorname{ch} \bar{C}+1)=(\operatorname{ch} B+1)(\operatorname{ch} D-1),
\end{array}
$$

в силу которых справедливы соответствующие формулы из (7), (8), (9), (10).

Для параллелограмма класса $h(I)$ согласно требованиям (23) имеем: $v>u, t u>u, t v>v, t v>t u$. Записывая данные неравенства для $t, u, v$, из (30) получаем:

$$
\operatorname{ch} \bar{A}<\operatorname{ch} B, \quad \operatorname{ch} \bar{A}<\operatorname{ch} D, \quad \operatorname{ch} B<\operatorname{ch} \bar{C}, \quad \operatorname{ch} D<\operatorname{ch} \bar{C} .
$$

Следовательно, справедливы неравенства из (7).

Для параллелограмма класса $h(I I)$ требования (23) не дают соответствующих неравенств между величинами гиперболических углов при вершинах.

Для параллелограмма класса $h(I I I)$ в силу требований (23) имеем: $v>u, t u<u, t v<v, t v>t u$. Записывая данные неравенства для $t, u, v$, из (32) получаем:

$$
\operatorname{ch} \bar{A}>\operatorname{ch} B, \quad \operatorname{ch} \bar{A}<\operatorname{ch} D, \quad \operatorname{ch} B<\operatorname{ch} \bar{C}, \quad \operatorname{ch} D>\operatorname{ch} \bar{C} .
$$

Следовательно, справедливы неравенства из (9).

Для параллелограмма класса $h(I V)$ согласно (23): $t u>u, t v<v$. Применив к данным неравенствам разложения (33), получаем:

$$
\operatorname{ch} \bar{A}<\operatorname{ch} D, \quad \operatorname{ch} B<\operatorname{ch} \bar{C} .
$$

Следовательно, справедливы неравенства из (10). Утверждение 3 теоремы доказано.

По формуле (4), учитывая условия (23), найдем выражения длин ребер параллелограмма $A B C D$ через параметры $t, u, v$ :

$$
\begin{array}{rlrl}
\operatorname{ch} \frac{|A B|}{\rho} & =\frac{2-u-v}{2 \sqrt{1-u} \sqrt{1-v}}, & \operatorname{ch} \frac{|B C|}{\rho}=\frac{2-v-t v}{2 \sqrt{1-v} \sqrt{1-t v}}, \\
\operatorname{ch} \frac{|C D|}{\rho}=\frac{2-t u-t v}{2 \sqrt{1-t u} \sqrt{1-t v}}, & \operatorname{ch} \frac{|A D|}{\rho}=\frac{2-u-t u}{2 \sqrt{1-u} \sqrt{1-t u}} .
\end{array}
$$

Запишем первое равенство из (34) в виде

$$
\left(\frac{\sqrt{1-u}}{\sqrt{1-v}}\right)^{2}-2 \frac{\sqrt{1-u}}{\sqrt{1-v}} \operatorname{ch} \frac{|A B|}{\rho}+1=0 .
$$

Для гиперболических параллелограммов каждого класса согласно требованиям из (23) $\sqrt{1-u}>\sqrt{1-v}$. Поэтому из квадратного относительно $(\sqrt{1-u}: \sqrt{1-v})$ уравнения (36) для гиперболических параллелограммов каждого класса получаем:

$$
\frac{1-u}{1-v}=e^{2 \frac{|A B|}{\rho}} .
$$

Согласно требованиям (23) выполняются соответственно неравенства

$$
\begin{array}{llll}
h(I): & 1-v>1-t v, & 1-t u>1-t v, & 1-u>1-t u \\
h(I I): & 1-v<1-t v, & 1-t u<1-t v, & 1-u>1-t u \\
h(I I I): & 1-v<1-t v, & 1-t u>1-t v, & 1-u<1-t u \\
h(I V): & 1-v<1-t v, & 1-t u>1-t v, & 1-u>1-t u
\end{array}
$$

Согласно условиям (38) из равенств (34), (35) получаем:

$$
h(I): \quad \frac{1-v}{1-t v}=e^{2 \frac{|B C|}{\rho}}, \quad \frac{1-t u}{1-t v}=e^{2 \frac{|C D|}{\rho}}, \quad \frac{1-u}{1-t u}=e^{2 \frac{|A D|}{\rho}} ;
$$




$$
\begin{aligned}
& h(I I): \quad \frac{1-v}{1-t v}=e^{-2 \frac{|B C|}{\rho}}, \quad \frac{1-t u}{1-t v}=e^{-2 \frac{|C D|}{\rho}}, \quad \frac{1-u}{1-t u}=e^{2 \frac{|A D|}{\rho}} ; \\
& h(I I I): \quad \frac{1-v}{1-t v}=e^{-2 \frac{|B C|}{\rho}}, \quad \frac{1-t u}{1-t v}=e^{2 \frac{|C D|}{\rho}}, \quad \frac{1-u}{1-t u}=e^{-2 \frac{|A D|}{\rho}} ; \\
& h(I V): \quad \frac{1-v}{1-t v}=e^{-2 \frac{|B C|}{\rho}}, \quad \frac{1-t u}{1-t v}=e^{2 \frac{|C D|}{\rho}}, \quad \frac{1-u}{1-t u}=e^{2 \frac{|A D|}{\rho}} .
\end{aligned}
$$

Подставляя соответствующие значения параметров $t, u, v$ из равенств (30)-(33) в выражения (37), (39)-(42), получим следующие соотношения:

$$
\begin{aligned}
& h(I): \quad e^{\frac{|A B|}{\rho}}=\operatorname{th} \frac{B}{2} \operatorname{cth} \frac{\bar{A}}{2}, \quad e^{\frac{|B C|}{\rho}}=\operatorname{th} \frac{\bar{C}}{2} \operatorname{cth} \frac{B}{2}, \\
& e^{\frac{|C D|}{\rho}}=\operatorname{th} \frac{\bar{C}}{2} \operatorname{cth} \frac{D}{2}, \quad e^{\frac{|A D|}{\rho}}=\operatorname{th} \frac{D}{2} \operatorname{cth} \frac{\bar{A}}{2} ; \\
& h(I I): \quad e^{\frac{|A B|}{\rho}}=\operatorname{cth} \frac{\bar{A}}{2} \operatorname{cth} \frac{B}{2}, \quad e^{\frac{|B C|}{\rho}}=\operatorname{cth} \frac{B}{2} \operatorname{cth} \frac{\bar{C}}{2}, \\
& e^{\frac{|C D|}{\rho}}=\operatorname{cth} \frac{\bar{C}}{2} \operatorname{cth} \frac{D}{2}, \quad e^{\frac{|A D|}{\rho}}=\operatorname{cth} \frac{\bar{A}}{2} \operatorname{cth} \frac{D}{2} ; \\
& h(I I I): \quad e^{\frac{|A B|}{\rho}}=\operatorname{th} \frac{\bar{A}}{2} \operatorname{cth} \frac{B}{2}, \quad e^{\frac{|B C|}{\rho}}=\operatorname{th} \frac{\bar{C}}{2} \operatorname{cth} \frac{B}{2}, \\
& e^{\frac{|C D|}{\rho}}=\operatorname{th} \frac{D}{2} \operatorname{cth} \frac{\bar{C}}{2}, \quad e^{\frac{|A D|}{\rho}}=\operatorname{th} \frac{D}{2} \operatorname{cth} \frac{\bar{A}}{2} ; \\
& h(I V): \quad e^{\frac{|A B|}{\rho}}=\operatorname{cth} \frac{\bar{A}}{2} \operatorname{cth} \frac{B}{2}, \quad e^{\frac{|B C|}{\rho}}=\operatorname{th} \frac{\bar{C}}{2} \operatorname{cth} \frac{B}{2}, \\
& e^{\frac{|C D|}{\rho}}=\operatorname{cth} \frac{\bar{C}}{2} \operatorname{cth} \frac{D}{2}, \quad e^{\frac{|A D|}{\rho}}=\operatorname{th} \frac{D}{2} \operatorname{cth} \frac{\bar{A}}{2} .
\end{aligned}
$$

На основании равенств (43)-(46) справедливы формулы (11)-(14). Теорема доказана.

Пусть в гиперболическом параллелограмме $A B C D$ на абсолюте правильно расположены точки соответствующей классу параллелограмма системы из (6). Тогда по утверждению 2 теоремы 1 точки $A, C(B, D)$ являются вершинами внутренних гиперболических псевдоуглов (углов) параллелограмма. Согласно формулам (11)-(14) для параллелограмма соответствующего класса имеют место равенства

$$
\begin{aligned}
& h(I): \quad|A B|+|B C|=|A D|+|C D| ; \quad h(I I): \quad|A B|+|C D|=|B C|+|A D| ; \\
& h(I I I): \quad|A B|+|A D|=|B C|+|C D| ; \quad h(I V): \quad|A B|=|B C|+|C D|+|A D| .
\end{aligned}
$$

Таким образом, следствиями теоремы 1 являются следующие утверждения.

Теорема 2. В параллелограмме класса $h(I)$ суммы длин ребер, исходящих из вершин внутренних гиперболических углов, равны.

Теорема 3. В параллелограмме класса $h(I I)$ суммы длин противоположных ребер равны.

Теорема 4. В параллелограмме класса $h(I I I)$ суммы длин ребер, исходящих из вершин внутренних гиперболических псевдоуглов, равны.

Теорема 5. В параллелограмме класса $h(I V)$ длина больщего ребра равна сумме длин трех других ребер параллелограмма.

По утверждению 1 теоремы 1 диагональная прямая $A C$ в параллелограмме $A B C D$ класса $h(I)$, $h(I I I), h(I V)$ может принадлежать каждому из трех типов прямых плоскости $\widehat{H}$. Тип прямой инвариантен во всех преобразованиях группы $G$. Таким образом, справедлива

Теорема 6. На плоскости $\widehat{H}$ все параллелограммы каждого из классов $h(I), h(I I I), h(I V)$ образуют по три инвариантных относительно группы $G$ рода.

В зависимости от типа диагональной прямой $A C$ (эллиптического $(e)$, гиперболического $(h)$, параболического $(p))$ параллелограммы классов $h(I), h(I I I), h(I V)$ отнесем соответственно к роду: $h(I) e$, $h(I) h, h(I) p ; h(I I I) e, h(I I I) h, h(I I I) p ; h(I V) e, h(I V) h, h(I V) p$. 


\section{Библиографический список}

1. Розенфельд Б. А. Неевклидовы пространства. М. Наука, 1969. 548 с.

2. Ромакина Л. Н. Простые разбиения гиперболической плоскости положительной кривизны // Мат. сб. 2012. Т. 203, вып. 9. С. 83-116.

3. Ромакина Л. Н. Овальные линии гиперболической плоскости положительной кривизны // Изв. Сарат. унта. Нов. сер. Сер. Математика. Механика. Информатика. 2012. Т. 12, вып. 3. С. 37-44.

4. Ромакина Л. Н. Аналоги формулы Лобачевского для угла параллельности на гиперболической плоскости положительной кривизны // Сиб. электрон. мат. изв. 2013. Т. 10. С. 393-407.
5. Ромакина Л. Н. Теорема о площади прямоугольного трехреберника гиперболической плоскости положительной кривизны // Дальневост. мат. журн. 2013. Т. 13, вып. 1. С. 127-147.

6. Ромакина Л. Н. Конечные замкнутые 3(4)-контуры расширенной гиперболической плоскости // Изв. Сарат. ун-та. Нов. сер. Сер. Математика. Механика. Информатика. 2010. Т. 1, вып. 3. С. 14-26.

7. Ромакина Л. Н. Конечные замкнутые 5-контуры расширенной гиперболической плоскости // Изв. Сарат. ун-та. Нов. сер. Сер. Математика. Механика. Информатика. 2011. Т. 11, вып. 1. С. 38-49.

\title{
Hyperbolic Parallelograms of the Plane $\widehat{H}$
}

\section{N. Romakina}

Saratov State University, Russia, 410012, Saratov, Astrahanskaya st., 83, romakinaln@mail.ru

Hyperbolic parallelograms on a Hyperbolic Plane $\widehat{H}$ of the positive curvature in the Cayley-Klein model are investigated. We conducted their classification, obtained the metric correlations between the measure of angles and the expressions of lengths of the edges through a measure of included angles.

Key words: hyperbolic plane $\widehat{H}$ of positive curvature; parallelogram; hyperbolic parallelogram.

\section{References}

1. Rozenfeld B. A. Neevklidovy prostranstva [Non-Euclidean spaces]. Moscow, Nauka, 1969, 548 p. (in Russian). 2. Romakina L. N. Simple partitions of a hyperbolic plane of positive curvature. Sb. Math., 2012, vol. 203, iss. 9. pp. 1310-1341.

3. Romakina L. N. Oval Lines of the Hyperbolic Plane of Positive Curvature. Izv. Sarat. Univ. N.S. Ser. Math. Mech. Inform., 2012, vol. 12, iss. 3, pp. 37-44 (in Russian).

4. Romakina L. N. Analogs of a formula of Lobachevsky for angle of parallelism on the hyperbolic plane of positive curvature. Siberian Electronic Mathematical Reports, 2013, vol. 10, pp. 393-407 (in Russian).
5. Romakina L. N. The theorem of the area of a rectangular trihedral of the hyperbolic plane of positive curvature. Far Eastern Mathematical Journal, 2013, vol. 13, № 1, pp. 127-147 (in Russian).

6. Romakina L. N. Finite Closed 3(4)-Loops of Extended Hyperbolic Plane. Izv. Sarat. Univ. N.S. Ser. Math. Mech. Inform., 2010, vol. 10, iss. 3, pp. 14-26 (in Russian).

7. Romakina L. N. Finite Closed 5-Loops of Extended Hyperbolic Plane. Izv. Sarat. Univ. N.S. Ser. Math. Mech. Inform., 2011, vol. 11, iss. 1, pp. 38-49 (in Russian).

УДК 517.54

\section{К РЕШЕНИЮ НЕОДНОРОДНОЙ КРАЕВОЙ ЗАДАЧИ ГИЛЬБЕРТА ДЛЯ АНАЛИТИЧЕСКОЙ ФУНКЦИИ В МНОГОСВЯЗНОЙ КРУГОВОЙ ОБЛАСТИ В ОСОБОМ СЛУЧАЕ}

\begin{abstract}
Р. Б. Салимов
Доктор фризико-математических наук, профрессор, заведующий касредрой высшей математики, Казанский государственный архитектурно-строительный университет, salimov@5354.ru

Предлагается новый подход к решению краевой задачи Гильберта для аналитической фрункции в многосвязной круговой области, основанный на построении решения соответствующей однородной задачи, когда определяется аналитическая в области функция по известным граничным значениям её аргумента. Рассматривается особый случай задачи, когда индекс задачи неотрицателен и меньше порядка связности области, уменьшенного на единицу. Картина разрешимости задачи зависит от разрешимости и числа решений соответствующей системы линейных алгебраических уравнений.

Ключевые слова: краевая задача Гильберта, индекс задачи, оператор Шварца.
\end{abstract}

